\title{
Análise contextual de reinternações frequentes de portador de transtorno mental ${ }^{*}$
}

\author{
Déborah Karollyne Ribeiro Ramos ${ }^{1}$ \\ Jacileide Guimarães ${ }^{2}$ \\ Bertha Cruz Enders ${ }^{3}$
}

RAMOS, D.K.R.; GUIMARÃES, J.; ENDERS, B, C. Contextual analysis of frequent hospital readmissions of the individual with mental disorder. Interface - Comunic., Saude, Educ., v.15, n.37, p.519-27, abr./jun. 2011.

This study analyzed the context of frequent hospital readmissions of individuals with psychic suffering in the following dimensions: immediate, specific, general and metacontextual. Following the contextual perspective proposed by the theoretical framework of Hinds, Chaves and Cypress (1992), the results of the narrative review of the scientific literature were categorized in four subthemes corresponding to the contextual dimensions: 1) the revolving door of psychiatry (immediate contextual level); 2) disarticulation of the mental health care network (specific contextual level); 3) the mad person and madness in the social mental imagery: the hospital as the "healing" place (general contextual level); 4) mental health paradigms: the epistemological dimension of psychiatric knowledge and action (metacontext). The analysis and comprehension of the contexts in which psychiatric hospital readmissions are included are significant so that the advances achieved by the Brazilian Psychiatric Reform and by the National Health System become effective.

Keywords: Mental disorder. Psychiatric hospital readmissions. Deinstitutionalization. Revolving door.
Este estudo analisou o contexto de reinternações frequentes de indivíduos com sofrimento psíquico em suas dimensões: imediata, específica, geral e metacontextual. Seguindo a perspectiva contextual proposta pelo referencial teórico de Hinds, Chaves e Cypress (1992), categorizaram-se os resultados encontrados na revisão narrativa da literatura científica em quatro subtemas referentes às dimensões contextuais: 1) a porta giratória da psiquiatria (nível contextual imediato); 2) desarticulação da rede de atenção em saúde mental (nível contextual específico); 3) o louco e a loucura no imaginário social: o hospital como o lugar da "cura" (nível contextual geral); 4) paradigmas da saúde mental: a dimensão epistemológica do saber/fazer psiquiátrico (metacontexto). Analisar e compreender os contextos nos quais estão inseridas as reinternações psiquiátricas torna-se, na atualidade, aspecto significativo para a efetivação dos avanços conquistados pela Reforma Psiquiátrica brasileira e pelo Sistema Único de Saúde.

Palavras-chave: Transtorno mental. Reinternação psiquiátrica. Desinstitucionalização. Porta giratória.
* Produção inédita resultante da disciplina "Análise crítica da prática de enfermagem" do Programa de Pós-graduação em Enfermagem da Universidade Federal do Rio Grande do Norte (PPGEnf-UFRN).

${ }^{1}$ Mestranda, Programa de Pós-graduação em Enfermagem, Departamento de Enfermagem, Universidade Federal do Rio Grande do Norte (PPGEnf-UFRN). Campus Universitário - $\mathrm{Br} 101$ Lagoa Nova, Natal, RN Brasil. 59.072-970 deborah_kr@hotmail.com 2,3 PPGEnf-UFRN 


\section{Introdução}

A Reforma Psiquiátrica brasileira pautada na desinstitucionalização do indivíduo com sofrimento psíquico, na redução progressiva de leitos psiquiátricos e na ênfase em tratamentos de base comunitária - compreendido como a garantia da livre circulação das pessoas com transtornos mentais pelos serviços, comunidade e cidade, além do oferecimento de cuidados com base nos recursos que a própria comunidade oferece -, trouxe novas concepções a respeito da "doença mental" e a desconstrução da ideia do hospital como o lugar da "cura" do louco e da loucura (Brasil, 2010).

A desinstitucionalização propõe a redução das internações psiquiátricas que, a partir de então, passam a se configurar como um instrumento clínico terapêutico indicado apenas em casos que exijam proteção da própria vida ou a de terceiros, devendo ser utilizada quando há ruptura no acompanhamento de base comunitária. A decisão do internamento deve ser tomada de forma criteriosa, especificamente em casos necessários, na tentativa de evitar a cronificação do indivíduo e da doença (Castro, 2009).

Para dar respaldo ao processo de redução de leitos hospitalares e do tempo de permanência nas instituições, faz-se necessária a construção de uma rede de alternativas à internação psiquiátrica, compatível com as necessidades dos pacientes que estão sendo devolvidos ao convívio familiar e social, para que tal retorno seja feito sem maiores traumas, tanto para o egresso quanto para o familiar cuidador (Parente et al., 2007).

A despeito das conquistas e dos avanços advindos da Reforma Psiquiátrica, são evidenciados, por meio de alguns estudos (Castro, 2009; Consoli, Hirdes, Costa, 2009; Parente et al., 2007; Alverga, Dimenstein, 2006; Bandeira, Barroso, 2005), altos índices de reinternações psiquiátricas. A "porta giratória" da psiquiatria, consoante Bandeira e Barroso (2005), configura-se como sendo um fenômeno caracterizado pelas reospitalizações frequentes dos portadores de transtornos mentais, obedecendo a um ciclo recidivo de internação/alta/internação.

A percepção de que a "porta giratória" é uma realidade presente nos serviços de saúde mental, tanto em nível local quanto em nível nacional, e que este fenômeno apresenta relevância e pertinência para os estudos acerca da rede de atenção em saúde/saúde mental, nos motivou a refletir acerca desse fenômeno, investigando sua dinâmica e inserção nos diversos níveis de contexto nos quais se desenvolve.

Assim, o objetivo deste artigo é analisar o fenômeno das reinternações frequentes de indivíduos com sofrimento psíquico nos níveis contextuais imediato, específico, geral e metacontextual. Utilizamos o referencial teórico de Hinds, Chaves e Cypress (1992), que dividem os níveis contextuais em quatro camadas interativas distintas entre si: o contexto imediato, o contexto específico, o contexto geral e o metacontexto. Vale ressaltar que entendemos os contextos como interligáveis e inter-relacionáveis, no entanto, para melhor compreensão didática, optamos por apresentá-los, neste artigo, seguindo a divisão proposta pelo referencial teórico.

O contexto imediato, como o próprio nome referenda, tem como característica principal a imediação, seu foco está no presente e é representado pelo fenômeno em si. O contexto específico é caracterizado pelo conhecimento individualizado e único que engloba o passado imediato e os aspectos relevantes da presente situação, em outras palavras, engloba os elementos que estão presentes no ambiente e influenciam o fenômeno. Os quadros de referência de vida do sujeito, desenvolvidos a partir de suas interpretações derivadas das interações passadas e atuais, constituem o contexto geral - nesta camada, crenças pessoais e valores culturais influenciam o fenômeno em questão. Finalmente, o metacontexto, reflete e incorpora o passado e o presente, além de destacar condições e aprendizados para o futuro (Hinds, Chaves, Cypress, 1992).

Seguindo a perspectiva contextual proposta pelo referencial teórico adotado, categorizamos os resultados encontrados na revisão narrativa da literatura científica em quatro subtemas, a saber: 1) a porta giratória da psiquiatria como o nível contextual imediato; 2) a desarticulação da rede de atenção à saúde mental como nível contextual específico; 3 ) o louco e a loucura no imaginário social: o hospital como o lugar da "cura" como nível contextual geral; 4) paradigmas da saúde mental: a dimensão epistemológica do saber/fazer psiquiátrico como o metacontexto. 


\section{A porta giratória da psiquiatria - contexto imediato do fenômeno das reinternações}

As novas concepções sobre saúde/doença mental transformaram os modelos de atenção destinados aos indivíduos com sofrimento psíquico. Nessa perspectiva, o modelo asilar/hospitalocêntrico vem sendo substituído por serviços e ações territoriais e comunitárias que potencializam o resgate da cidadania do paciente psiquiátrico egresso daqueles hospitais. Por outro lado, todas as metamorfoses decorrentes dos movimentos da Reforma Psiquiátrica suscitaram desafios para os cuidadores e trabalhadores da saúde mental. Para Gastal et al. (2000), uma das principais dificuldades resultantes dessa transição paradigmática é o paciente de revolving door, ou seja, os usuários das políticas de saúde mental da atualidade, sujeitos ao fenômeno da porta giratória.

Revolving door é uma expressão inglesa utilizada para o termo porta giratória (Rotelli, Leonardis, Mauri, 2001). Esse fenômeno vem sendo estudado desde a década de 1960 e configura-se como importante aspecto do atendimento psiquiátrico não anteriormente planejado, mas que surgiu como consequência da mudança do modelo hospitalocêntrico (Gastal et al., 2000).

Sabe-se que o paciente de revolving door é aquele admitido e liberado frequentemente no hospital psiquiátrico, porém ainda não existe um consenso entre os autores a respeito da periodicidade dessas readmissões constantes, havendo uma variação na literatura que refere os seguintes parâmetros: entre três ou mais admissões em um período de dois anos; quatro ou mais em um período de cinco anos; quatro internações ou mais sem intervalo superior a dois anos e meio, em um período de dez anos; ou, ainda, quatro ou mais em um período de cinco anos (Parente et al., 2007).

Levando em consideração o número de internações e a quantidade de dias de reclusão, atentamos para a grande problemática que envolve as reinternações frequentes de pacientes psiquiátricos. Experiências repetitivas de reclusão podem desencadear rupturas nos laços familiares e na permanência desses indivíduos no seu entorno social; conforme Salles e Barros (2007, p.74), "durante a internação se desaprendem as regras básicas de convivência e ao sair, a pessoa fica marcada pelo estigma e preconceito".

Castro (2009) sugere que a porta giratória ocorre com mais frequência em pacientes crônicos, com maiores comprometimentos e maior tempo de permanência em instituições psiquiátricas hospitalares. Em contrapartida, o autor aponta para a mudança de perfil dos pacientes que estão sendo admitidos, sobretudo, devido: ao aumento da complexidade dos casos, à resistência aos diversos tipos de tratamento, ao aumento de internações por mandatos judiciais e ao aumento no número de primeiras internações.

Essa primeira aproximação do nível contextual, referente à porta giratória no fenômeno das reinternações psiquiátricas, é particularmente relevante se considerarmos os demais níveis contextuais na busca do desafio da efetivação teórico-prática da assistência em saúde mental à luz da premissa da qualidade do acesso e do acompanhamento/tratamento no âmbito da atualidade das políticas públicas de saúde mental, notadamente, dos princípios e diretrizes do Sistema Único de Saúde e da Reforma Psiquiátrica brasileira.

\section{Desarticulação da rede de atenção à saúde mental - contexto específico das reinternações psiquiátricas}

A rede de atenção à saúde mental no Brasil preconiza uma série de serviços que devem funcionar de forma articulada para maximizar a autonomia e a cidadania da pessoa com sofrimento psíquico, especialmente, aquelas egressas de hospital psiquiátrico, assim como, reduzir o índice de primeiras internações e/ou reinternações psiquiátricas.

Atualmente, a rede de serviços e equipamentos em saúde mental dispõe de: Centros de Atenção Psicossocial (CAPS I e II, CAPS III, CAPSad, CAPSi); Serviços Residenciais Terapêuticos (SRT), subsidiados pelo Programa De volta para casa, que oferece auxílio financeiro para egressos de longas internações em hospitais psiquiátricos; Centros de Convivência e Cultura; leitos de atenção integral em Hospitais Gerais e nos CAPS III; Programa Nacional de Avaliação dos Serviços Hospitalares e Psiquiatria; Programa Permanente para Formação de Recursos Humanos para a Reforma Psiquiátrica; 
Supervisão Clínico-Institucional dos CAPS; Programa Nacional de Avaliação; a estratégia de Apoio Matricial em Saúde Mental, e os Núcleos de Apoio em Saúde da Família-NASF (Brasil, 2010).

A rede, portanto, é constituída por serviços, políticas e estratégias, intenções e gestos fundamentais para a exequibilidade da Reforma Psiquiátrica brasileira, e, consequentemente, para a realização de um processo de desinstitucionalização efetivo do indivíduo egresso de instituição psiquiátrica hospitalar, assim como, para o fomento de ações de promoção da saúde, prevenção e intervenção precoce em saúde mental, buscando o cumprimento da meta de internação como último recurso terapêutico, quando nenhuma outra estratégia alcançou resolubilidade da situação vivenciada, evitando, portanto, o fenômeno da (re)internação psiquiátrica, historicamente associada à pouca resolubilidade e alta cronificação da condição da pessoa internada. A articulação entre os serviços da rede de atenção em saúde mental com os demais serviços e equipamentos de saúde e com os demais setores da sociedade compõe o requisito da intersetorialidade dentro e fora do setor saúde; isso contribui, significativamente, para a conquista mais ampla da atenção psicossocial e da inclusão do paciente psiquiátrico por meio da redução do dispositivo da reinternação psiquiátrica.

O que se entende por articulação da rede de atenção à saúde mental? Consoli, Hirdes e Costa (2009) relacionam uma série de ações que, de forma contígua, favorece a articulação da rede de atenção, destacando-se: a gestão competente dos recursos destinados à saúde mental, a quantidade suficiente de serviços substitutivos, o número de leitos psiquiátricos condizentes com o preconizado pela Reforma Psiquiátrica brasileira, além do bom funcionamento do sistema de referência e contrarreferência.

Outro fator intrínseco ao bom êxito do funcionamento da rede de atenção é a realização de ações territoriais expressivas e impactantes, desenvolvidas, especialmente, pelo apoio matricial e/ou pelo CAPS, uma vez que, conforme Quintas e Amarante (2008), a proximidade entre este serviço e a comunidade favorece o conhecimento das individualidades e das peculiaridades de cada usuário, o que, consequentemente, facilita a promoção da autonomia dos indivíduos com sofrimento psíquico e seu engajamento no contexto social do qual fazem parte.

A acessibilidade, a diversificação das ações, a existência de profissionais qualificados para atender à demanda em saúde mental, e a responsabilidade com a desmistificação/desconstrução do imaginário social acerca do ser louco e da loucura, são outras características de uma rede de atenção articulada (Alverga, Dimenstein, 2006). A articulação desses serviços, equipamentos, saberes e práticas, cada um desempenhando adequadamente sua função dentro do contexto da Reforma Psiquiátrica, contribui para o resgate pessoal e social do usuário na rede de atenção, tanto em processo de desinstitucionalização quanto em fase aguda das psicopatologias, conjuntamente com os profissionais de saúde, a família e a sociedade em geral.

Os princípios da desinstitucionalização dos cuidados em saúde mental - tais como a formação de uma equipe multiprofissional, a integralidade na assistência, a corresponsabilidade da equipe vinculada ao território, portanto, a base comunitária, a intersetorialidade e o enfoque interinstitucional respaldam e operacionalizam o apoio matricial que constrói a articulação da saúde mental com a saúde da família, prerrogativa almejada e necessária para se atender à demanda de suporte aos usuários dos serviços e seus familiares (Vecchia, Martins, 2009).

Para Pitiá e Furegato (2009), quando as equipes de saúde da família se responsabilizam pelo acompanhamento adequado ao indivíduo com sofrimento psíquico severo, podem ser observados resultados positivos, como: a diminuição no número de reinternações psiquiátricas e a promoção da saúde mental e reintegração social para pacientes psicóticos.

Cientes da interdependência entre a efetivação dos dispositivos da reforma psiquiátrica, o êxito da desinstitucionalização, a conquista da redução de internações psiquiátricas e a resolubilidade da articulação da rede de atenção à saúde mental, pressupomos que há relação direta entre o fenômeno de reinternações psiquiátricas (revolving door ou porta giratória) e a desarticulação ou pouca articulação da rede de atenção à saúde mental, apresentando-se, no nível contextual específico, como um indicativo da necessidade premente de organização através da intersetorialidade, dentro e fora do setor saúde, para a consolidação dos avanços e o enfrentamento dos novos desafios trazidos pelo processo de desinstitucionalização em saúde mental. 


\section{O louco e a loucura no imaginário social: o hospital como o lugar da "cura" - contexto geral das reinternações psiquiátricas}

Desde os primórdios da civilização humana, têm-se relatos de indivíduos portando sintomas psicóticos. As formas de atenção e os níveis de aceitação social dos "loucos" foram se transformando ao longo do tempo, mediante o contexto histórico no qual estavam inseridos.

$\mathrm{Na}$ Antiguidade, acreditava-se na interferência de entidades sobrenaturais sobre a razão humana, ou seja, os povos entendiam a diferença da razão predominante como um capricho ou um castigo dos Deuses. Já no período hipocrático, o fenômeno da loucura adquire um caráter organicista, no qual era concebida como um desarranjo na natureza orgânica do homem. Na Idade Média, surge o enfoque demonista, período em que a Igreja católica imperou no tratamento do "mal da loucura" através de exorcismos e orações. Por volta de 1800, Pinel destaca-se atribuindo um caráter moral à doença mental, instituindo, assim, o Tratamento Moral, em que a alienação predominante era fruto de causas orgânicas e morais. Assim, o indivíduo deveria receber um tratamento de cunho moral por meio de uma reeducação pedagógica afastada do convívio familiar e das influências sociais (Silva, Zanello, 2010).

Neste cenário, o hospital passou a simbolizar o espaço de observação e de "cura" para o alienado. Este ideal de tratamento recluso e disciplinador perdurou no Brasil por cerca de duzentos anos, até a efervescência de movimentos que denunciavam a segregação e os maus-tratos sofridos por pessoas com transtornos mentais internadas em instituições psiquiátricas. No entanto, a despeito das mudanças desencadeadas pela Reforma Psiquiátrica brasileira, as prerrogativas herdadas por cerca de dois séculos de uma cultura hospitalocêntrica permanecem arraigadas no cotidiano de profissionais e serviços, dentro e fora do setor saúde, orientando ações, estigmatizando e rejeitando a diferença trazida pelo sofrimento psíquico como sinônimo exclusivo de incapacidade e periculosidade.

Para Amarante (2009), as representações sociais agregam a diferença e a divergência dos indivíduos em sofrimento psíquico à incapacidade social e à impossibilidade de estabelecerem trocas sociais. Desse modo, as estratégias de intervenção, no campo cultural, devem objetivar transformar o lugar da loucura no imaginário social. Destacamos o agravante de que o campo cultural e sua herança hospitalocêntrica impactam o julgamento não só de leigos e da sociedade em geral, mas também do próprio setor saúde e, até, de segmentos dentro da própria área de assistência em saúde mental. Essa constatação histórica e institucional demarca uma maior dificuldade para a apreensão de novos saberes e práticas e, consequentemente, para a exequibilidade da rede de atenção e para o substrato, não de cura, herdado das ciências exatas, mas a noção de convivência propugnada pela Reforma Psiquiatria brasileira como fator desinstitucionalizante e propiciador da autonomia da pessoa com sofrimento psíquico.

Diante do exposto, acreditamos que crenças acerca do saber/fazer psiquiátrico contribuem para as reinternações frequentes de indivíduos em sofrimento psíquico. Cardoso e Galera (2009) afirmam que o fenômeno das reinternações psiquiátricas está permeado por representações social, cultural e econômica, tanto dos profissionais que decidem por esse procedimento, quanto dos familiares e sociedade em geral.

As sensações de tranquilidade e alívio, experimentadas pelo familiar devido à internação da pessoa com sintomatologia psiquiátrica presente é um fator impactante na preferência de familiares pela transferência total da responsabilidade do cuidado para o hospital, evidenciando a fragilidade do conceito de "cura" da doença mental, que atribui um caráter natural à ruptura dos laços familiares e do convívio social provocados pela internação (Cardoso, Galera, 2009). Isso porque, constata-se, na atualidade, que o isolamento familiar e social da pessoa com sofrimento psíquico, através de longas internações em hospitais psiquiátricos, exacerbou a condição do doente e da doença, contribuindo para o que ficou conhecido como a cronificação da pessoa e da doença - fato desabonador para a condição humana relegada ao esquecimento travestido em um diagnóstico-rótulo que, historicamente, contribuiu para a eleição do objeto patológico em detrimento do sujeito em experiência de sofrimento. Com isso, tem-se a consequência do desejo veemente da cura distante em detrimento da custosa convivência. 
Com a efetivação da rede de atenção, especialmente, por intermédio de ações territoriais no dia a dia de famílias com indivíduos em sofrimento psíquico, as relações seriam menos traumáticas, os cuidadores saberiam lidar melhor com o indivíduo com transtorno mental, evitando recorrer ao hospital, podendo a ideia do hospital como o lugar da "cura" do louco ser desmistificada à luz da compreensão histórica de abusos cometidos em nome de terapêuticas psiquiátricas. No entanto, de acordo com Pitiá e Fugerato (2009), este processo de articulação exige mais do que a criação de serviços substitutivos, a regulamentação das equipes de saúde mental ou a normatização das ações estratégicas. É imprescindível, portanto, a desconstrução de concepções manicomiais, arraigadas em mentalidades de profissionais e familiares, o que influencia, imensamente, a qualidade do atendimento aos indivíduos com sofrimento psíquico.

\section{Paradigmas da saúde mental: a dimensão epistemológica do saber/fazer psiquiátrico - metacontexto das reinternações psiquiátricas}

Durante muito tempo, a psiquiatria foi norteada pelo positivismo e o cartesianismo. Sob tal orientação, foram priorizadas ações centradas na doença reduzida a uma alteração biológica; o hospital e o saber médico-psiquiátrico foram instituídos, respectivamente, como o lugar e o discurso competentes, fortalecidos pela adesão à terapêutica de reclusão. Essa forma de perceber e orientar as ações da comunidade científica da área tornou-se reconhecida como o paradigma hospitalocêntrico medicalizador (Amarante, 2009). Segundo Khun (1969), paradigma é o conjunto de elementos culturais, conhecimentos e códigos teóricos, técnicos ou metodológicos, compartilhados pelos membros de uma comunidade científica, assim, pretensamente, distinguindo-a da crença ou do senso comum.

Desta forma, guiada por uma visão biologicista, a psiquiatria desenvolveu uma assistência voltada para o quadro sintomatológico, marcada pela alienação social do sujeito, segregação, medicalização, contenção física e química, com caráter custodial e disciplinante, em que o indivíduo é o objeto do tratamento (Pitiá, Furegato, 2009).

A eclosão dos movimentos da Reforma Psiquiátrica brasileira suscitou novas discussões, especialmente no que se refere à dimensão epistemológica da psiquiatria, ou seja, no campo teóricoconceitual que fundamenta e autoriza o saber/fazer médico-psiquiátrico. Nesse cenário, os principais conceitos epistêmicos da psiquiatria - tais como alienação/doença mental, isolamento terapêutico, cura, internação - são contestados e, a partir da óptica do pensamento complexo, reavaliados e desconstruídos, isto é, refletidos, repensados à luz da própria história da área (Amarante, 2009).

Toda esta transformação epistemológica precipitou uma crise no paradigma hospitalocêntrico, fazendo emergir novas buscas para o pensar e o agir em psiquiatria/saúde mental. Assim, surge o paradigma psicossocial caracterizado pelo envolvimento do sujeito no seu próprio tratamento e pela desconstrução do arcabouço teórico da psiquiatria clássica, objetivando transformar a lógica organizacional dos serviços de saúde mental e criar uma rede de atenção substitutiva ao hospital e pautada na atenção territorial e inclusiva da pessoa com sofrimento psíquico.

Sob a égide desse novo paradigma, as ações desenvolvidas revestem-se de caráter social: ao sujeito estima-se o respeito à sua subjetividade, destaca-se a ênfase na multiprofissionalidade/ interdisciplinaridade e na importância do convívio familiar. O atendimento hospitalar passa a ter a função restrita de impacto em situações de crise, orientado pelo princípio do retorno rápido ao convívio em sociedade (Pitiá, Furegato, 2009). Em nível mais amplo, evidencia-se a criação de portarias ministeriais e políticas públicas voltadas para a defesa da saúde e da cidadania do indivíduo com sofrimento psíquico.

A despeito das transformações advindas da Reforma Psiquiátrica brasileira, o metacontexto do fenômeno das reinternações psiquiátricas demonstra a coexistência dos paradigmas hospitalocêntrico e psicossocial no saber/fazer da saúde mental, onde predominam, em discursos e políticas oficiais, esforços para a efetivação da rede de atenção que reduziria a porta giratória de internação/alta/ reinternação; e, na resolubilidade atual da mesma rede, ainda prevalece o gesto contraditório de reinternações psiquiátricas, reiterado por insuficiência quantitativa de serviços e qualitativa da 
formação dos profissionais, fato que, segundo Rinaldi e Bursztyn (2008), desafia a perspectiva de uma atenção psicossocial e uma prática clínica manejada por uma equipe multiprofissional.

Profissionais e serviços buscam realizar as propostas ministeriais que regulamentam a inclusão social e o mínimo necessário de internação psiquiátrica, no entanto, muitos profissionais e serviços substitutivos também estão impregnados pela lógica manicomial, fato que alimenta, consideravelmente, os índices, ainda preocupantes, de reinternações psiquiátricas no âmbito da Reforma Psiquiátrica brasileira.

O contraste da transição paradigmática, em que dimensões epistemológicas se confundem e coexistem, identifica o metacontexto do fenômeno das reinternações psiquiátricas como um campo onde há evidentes resquícios da prática hospitalocêntrica com forte ideologia segregadora e estigmatizante. Em contrapartida, têm-se as prerrogativas do Sistema Único de Saúde (SUS), mais especificamente, da Política Nacional de Atenção à Saúde Mental, através da rede de atenção preconizada pela Reforma Psiquiátrica brasileira. O discurso e os esforços por uma rede de atenção em saúde mental endossam avanços e conquistas por uma atenção psicossocial, cujo maior desafio revela-se na incidência e na prevalência do fenômeno da porta giratória ou reinternações psiquiátricas.

\section{Considerações finais}

As reinternações psiquiátricas configuram-se como um dado significativo e preocupante no que se refere à demonstração da efetividade e resolubilidade da rede de atenção em saúde mental.

Analisar os níveis contextuais da porta giratória em psiquiatria torna-se pertinente e relevante para a compreensão e intervenção junto à atualidade dos saberes e práticas da saúde mental no Brasil, uma vez que abordamos, gradativamente, aspectos imediatamente relacionados ao fenômeno em análise, bem como aspectos específicos e gerais, obtendo-se uma síntese simultaneamente circunstanciada e panorâmica da problemática em questão.

Assim, esperamos contribuir para a compreensão e resolubilidade das transformações no âmbito dos saberes e práticas da saúde mental brasileira, especialmente, no tocante à desconstrução do aparato manicomial resistente à efetivação da rede de atenção em saúde mental intersetorial.

A ênfase intersetorial justifica-se pela necessidade premente de engajamento e articulação dos saberes e práticas, tanto do próprio setor saúde, quanto das demais áreas da sociedade - a exemplo do debate e das intenções da IV Conferência Nacional de Saúde Mental, realizada em junho de 2010, que envolveu temas relevantes para o campo da saúde mental, na perspectiva da intersetorialidade, e promoveu deliberações acerca dos desafios para a melhoria dos cuidados em saúde mental no território, além de contemplar o desenvolvimento de ações intersetoriais para o fortalecimento das ações em saúde mental nesse novo cenário de Reforma Psiquiátrica (Brasil, 2010).

\section{Colaboradores}

Déborah Karollyne Ribeiro Ramos foi responsável pela idealização, busca bibliográfica e redação do manuscrito; Jacileide Guimarães, pela revisão final, realizando contribuições significativas acerca dos constructos teóricos; Bertha Cruz Enders colaborou, ao longo da disciplina "Análise crítica da prática de Enfermagem", na idealização do contexto como foco de análise e na construção do conhecimento que possibilitou a elaboração do manuscrito.

\section{Referências}

ALVERGA, A.R.; DIMENSTEIN, M. A reforma psiquiátrica e os desafios na desinstitucionalização da loucura. Interface - Comun., Saude, Educ., v.10, n.20, p.299-316, 2006. 
AMARANTE, P. Reforma psiquiátrica e epistemologia. Cad. Bras. Saude Mental, v.1, n.1, p.1-7, 2009. Disponível em: <http://www.abrasme.org.br/cbsm/artigos/artigos/04_ Paulo_Amarante.pdf>. Acesso em: 23 nov. 2010.

BANDEIRA, M.; BARROSO, S.M. Sobrecarga de pacientes psiquiátricos. J. Bras. Psiquiatr., v.54, n.1, p.34-46, 2005.

BRASIL. Ministério da Saúde. Regimento da IV Conferência Nacional de Saúde Mental - Intersetorial. Brasília, 2010. Disponível em: < http://portal.saude.gov.br/portal/ arquivos/pdf/regimento_interno_conferencia_02_03.pdf>. Acesso em: 23 nov. 2010.

CARDOSO, L.; GALERA, S.A.F. Quem são os egressos de internação psiquiátrica? Acta Paul. Enferm., v.22, n.6, p.733-40, 2009.

CASTRO, S.A. Caracterização sociodemográfica e clínica das reinternações psiquiátricas no Hospital Santa Tereza de Ribeirão Preto, no período de 2006 a 2007. 2009. Dissertação (Mestrado) - Escola de Enfermagem de Ribeirão Preto, Universidade de São Paulo, Ribeirão Preto. 2009.

CONSOLI, G.L.; HIRDES, A.; COSTA, J.S.D. Saúde mental nos municípios do Alto Uruguai, RS, Brasil: um diagnóstico da reforma psiquiátrica. Cienc. Saude Colet., v.14, n.1, p.117-28, 2009.

GASTAL, L. G. et al. Predicting the revolving door phenomenon among patients with schizophrenic, affective disorders and non-organic psychoses. Rev. Saude Publica, v.34, n.3, p.280-5, 2000.

HINDS, P.; CHAVES, D.E.; CYPRESS, S.M. Context as a source of meaning and understanding. Qual. Health Res., v.2, n.1, p.61-74, 1992.

KUHN, T.A. A estrutura das revoluções científicas. São Paulo: Perspectiva, 1970.

PARENTE, C.J.S. et al. O fenômeno do revolving door em hospitais psiquiátricos de uma capital do Nordeste brasileiro. REME - Rev. Min. Enferm., v.11, n.4, p.381-6, 2007.

PITIÁ, A.C.A.; FUREGATO, A.R.F. O Acompanhamento Terapêutico (AT): dispositivo de atenção psicossocial em saúde mental. Interface - Comun., Saude, Educ., v.13, n.30, p.67-77, 2009. Disponível em: <http://www.interface.org.br/arquivos/aprovados/ artigo133.pdf>. Acesso em 23 nov. 2010.

QUINTAS, R.M.; AMARANTE, P. A ação territorial do centro de atenção psicossocial em sua natureza substitutiva. Saude Debate, v.32, n.78-80, p.99-107, 2008.

RINALDI, D.L.; BURSZTYN, D.C. O desafio da clínica na atenção psicossocial. Arq. Bras. Psicol., v.60, n.2, 2008. Disponível em: <http://146.164.3.26/seer/lab19/ojs2/index. php/ojs2/article/viewArticle/243/182>. Acesso em: 23 nov. 2010.

ROTELLI, F.; LEONARDIS, O.; MAURI, D. Desinstitucionalização, uma outra via. In: NICÁCIO, F. (Org.). Desinstitucionalização. 2.ed. São Paulo: Hucitec, 2001. p.17-59.

SALLES, M.M.; BARROS, S. Reinternação em hospital psiquiátrico: a compreensão do processo saúde/doença na vivência do cotidiano. Rev. Esc. Enferm. USP, v.41, n.1, p.73-81, 2007.

SILVA, M.; ZANELLO, V.M. Religiosidade e loucura: a influência da religião na forma como o "doente mental" enfrenta a doença. Psicol. IESB, v.2, n.1, p.37-47, 2010.

VECCHIA, M.D.; MARTINS, S.T.F. De-institutionalization of care for people with mental disorders within primary healthcare settings: contributions towards implementation of actions. Interface - Comunic., Saude, Educ., v.13, n.28, p.151-64, 2009. 
RAMOS, D.K.R.; GUIMARÃES, J.; ENDERS, B, C. Análisis contextual de reingresos frecuentes del portador de trastorno mental. Interface - Comunic., Saude, Educ., v.15, n.37, p.519-27, abr./jun. 2011.

Este estudio examina el contexto de los frecuentes reingresos de pacientes con trastornos psicológicos en las dimensiones: inmediatas, específicas, generales y meta-contextuales. Siguiendo la perspectiva contextual propuesta por el marco teórico de Hindes, Chaves y Cypress (1992), los resultados encontrados en la revisión narrativa de la literatura científica se clasifican en cuatro sub-temas relativos a las dimensiones contextuales: 1) la puerta giratoria de la psiquiatría (contexto inmediato); 2) desarticulación de la red de salud mental (contexto específico); 3) el loco y la locura en lo imaginario social; el hospital como un lugar de "curación" (contexto general); 4) paradigmas de salud mental: la dimensión epistemológica del saber/hacer psiquiátricos (metacontexto). Analizar y comprender los contextos donde se insertan los reingresos psiquiátricos se convierte, actualmente, en un aspecto significativo para confirmar los avances conquistados por la Reforma Psiquiátrica brasileña y el Sistema Único de Salud.

Palabras clave: Trastorno mental. Reingresos psiquiátricos. Desinstitucionalización. Puerta giratoria.

Recebido em 06/09/2010. Aprovado em 18/11/2010 
